\title{
Influence of monitoring duration on measured traffic action effects on road bridges
}

B. Sawicki \& E. Brühwiler

Laboratory of Maintenance and Safety of Structures (MCS), École Polytechnique Fédérale de Lausanne
(EPFL), CH-1015, Lausanne, Switzerland

\section{ABSTRACT}

Direct monitoring of action effects is part of a novel method for safety verification of structures. When Level I assessment using code provisions and load models shows that the minimum required safety level of structure is not achieved, Level II verification comprising measurements of structural response under traffic action should be used. The duration of monitoring largely influences the obtained results for both the Fatigue and Ultimate Limit State verifications.

This paper analyses the influence of monitoring duration on the obtained results. Data from two long term (2.5 years) monitoring campaigns is resampled (bootstrapped) and analyzed to explore the stochastic nature of traffic. The Extreme Value Theory and the Narrowing Confidence Interval method serve to answer what is the required monitoring duration to achieve reliable results. Furthermore, the Cumulative Damage correction Factor is proposed. This monitoring duration dependent $\gamma_{\mathrm{CDF}}$ factor is to be used for the extrapolation of fatigue damage registered during monitoring campaign. It takes into account lack of representability of data due to observation period and stochastic nature of road traffic.

Two road bridges monitored with strain gauges and with different nature of traffic are considered: (a) a heavily loaded two-lane highway viaduct and (b) a state road viaduct with a bi-directional traffic; thus the obtained results can be generalized.

The main conclusions are that the monitoring duration of road bridge should not be shorter than 100 days. Due to variation of the structural response due to the asphalt pavement depending on the ambient temperature, the monitoring should be executed during hot months to be conservative. The cumulative damage obtained in one-year-long monitoring campaign should be multiplied with factor $\gamma_{\mathrm{CDF}}=1.3$ (or $\gamma_{\mathrm{CDF}}=2.5$ ) to take into account possible favorable arrangement of traffic during the measurements, before extrapolation to the total service duration of bridge is done.

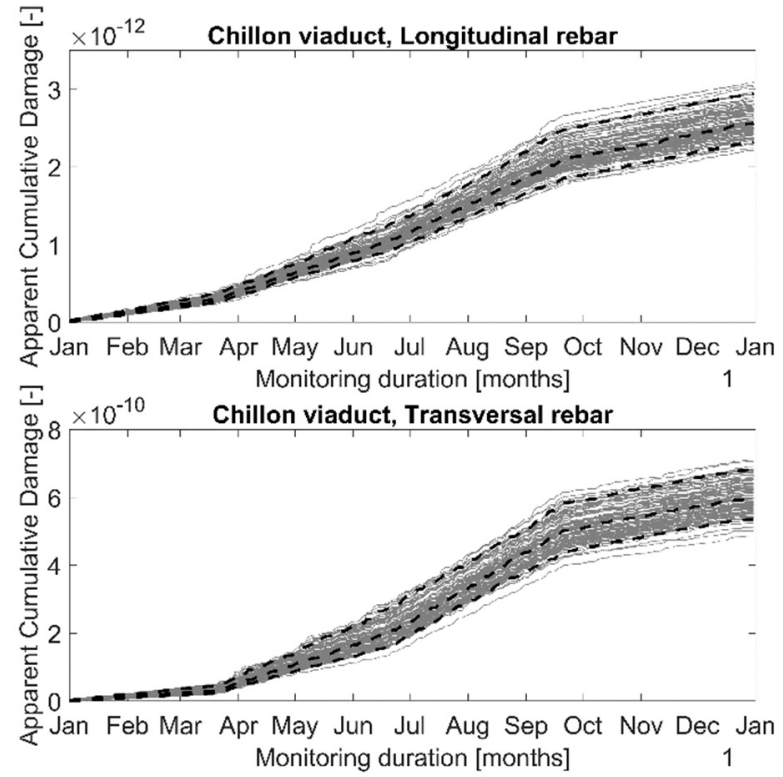

Figure 1. Resampled apparent cumulative damage for two strain gauges of Chillon viaducts. Dashed curves represent $5^{\text {th }}, 50^{\text {th }}$ and $95^{\text {th }}$ fractiles.

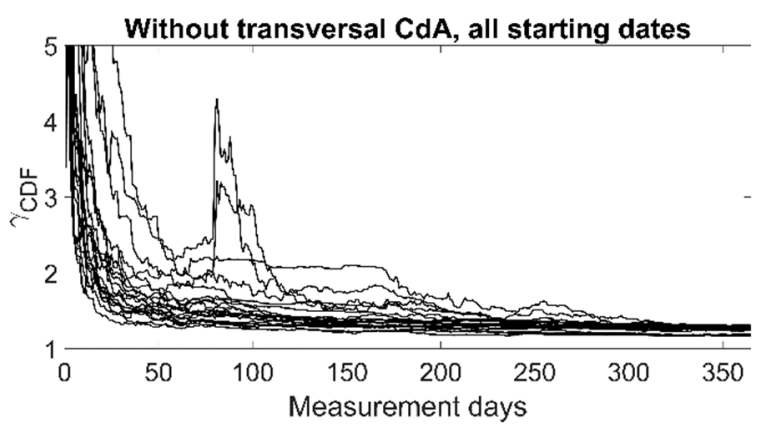

Figure 2. Cumulative Damage correction Factor $\gamma_{\mathrm{CDF}}$ based on five gauges (not taking into account transversal rebars of Crêt de l'Anneau) and four starting dates. 